\title{
Identification of elasticity modulus by vibratory analysis (Application to a natural composite: Aleppo pine wood)
}

\section{Identification des modules d'élasticité par analyse vibratoire (Application à un composite naturel: bois de Pin d'Alep)}

\author{
Abdelhakim DAOUI ${ }^{1,2}$, Abdellatif ZERIZER ${ }^{2}$ \\ ${ }^{1}$ Département Physique, Faculté des Sciences, Université M'hamed Bougara Boumerdes, Algérie \\ ${ }^{2}$ UR-MPE Unité de Recherche Matériaux Procédé Environnement, Université Bougara, Boumerdès, Algérie
}

\begin{abstract}
In this paper, we present a method for the determination of the elasticity modulus in the vibratory domain of materials. This approach is based on research and interpretation of the spectrum of natural frequencies resulting from natural vibrations based on the theory of elastic beams. The tests consist of classical tests of longitudinal vibrations of natural composite beams (Aleppo pine wood), long enough to observe some natural frequencies. This identification method showed a good correlation between the theoretical and experimental values, notably the evaluation of the modal parameter for the case of the resonant frequencies and the identification of the modulus of elasticity of the materials used.
\end{abstract}

Résumé. Dans ce papier, nous présentons une méthode pour la détermination du module d'élasticité dans le domaine vibratoire des matériaux. Cette démarche repose sur la recherche et l'interprétation du spectre des fréquences propres issues des vibrations naturelles sur la base de la théorie des poutres élastiques. Les essais consistent en des tests classiques de vibrations longitudinales de poutres composites naturelles (bois de Pin d'Alep), suffisamment longues pour observer quelques fréquences propres. Cette méthode d'identification a montré une bonne corrélation entre les valeurs théoriques et expérimentales, notamment l'évaluation du paramètre modale pour le cas des fréquences de résonance et l'identification du module d'élasticité des matériaux utilisé.

Mots Clés: analyse vibratoire, acquisition de données, module d'élasticité, fréquence de résonance, théorie des poutres élastiques

\section{Introduction}

Les essais d'évaluation non destructive vibratoire sont basés sur l'analyse spectrale des vibrations naturelles d'un élément de structure (poutre, panneau) soumis à une excitation impulsionnelle. Cette méthode, applicable à bien des matériaux, donne une estimation instantanée des propriétés mécaniques des matériaux testés et permet d'établir une identification mécanique de chaque pièce (Haines and Leban, 1997; Zaveri et Phil, 1984). Les avantages de cette méthodologie, outre le fait qu'elle soit non-destructive, reposent sur sa simplicité, sa répétitivité et traçabilité.

Les données issues de ces essais permettront d'orienter les choix des matériaux de fabrication, d'optimiser les critères relatifs aux propriétés mécaniques, et bien sur d'accroître l'efficacité du classement en se substituant au classement visuel.

Dans les analyses vibratoires, on est toujours amené vers la mesure des modes de vibration (fréquences naturelles 
de vibration) afin de les comparer à ceux obtenus par des méthodes théoriques. On estime que la confirmation des modes propres de vibration par des essais peut fournir la réassurance pour la validation des résultats des essais effectués (Ewins, 1984).

\section{Définition de la méthode d'analyse vibratoire}

\subsection{Mécanisme d'excitation de structures et d'acquisition de données}

La méthode d'analyse vibratoire la plus couramment utilisée dans le domaine expérimental consiste en l'excitation de la structure et l'enregistrement de sa réponse. Les techniques de mesure des sollicitations exercées sur les structures et les réponses de ces dernières pour l'analyse dynamique comprennent trois aspects fondamentaux (DØssing, 1987a; DØssing, 1988b; Dumas et Bennevault, 2001) :

- Le mécanisme permettant d'exciter correctement la structure

- Celui permettant de mesurer les signaux souhaités (forces, accélérations, déplacement...etc.)

- Et enfin celui qui assure l'extraction des informations que l'on désire acquérir.

Les excitateurs peuvent être classés en deux catégories : ceux directement en contact avec la structure étudiée pendant toute la durée de l'essai et ceux qui agissent à distance ou qui ne sont en contact que pendant un certain laps de temps (cas des marteaux d'impulsion pour le cas de test d'excitation transitoire).

\subsection{Transformée de Fourier Discrète d'une fonction réelle}

Le calcul et la représentation des fonctions nécessitent la connaissance des signaux dans le domaine fréquentiel. En pratique, on a seulement accès aux signaux dans le domaine temporel (réponse d'un accéléromètre par exemple). Le passage du domaine temporel au domaine fréquentiel est réalisé à l'aide de la Transformée de Fourier Rapide ( FFT).

L'expression de la FFT d'une fonction réelle $\mathrm{y}(t)$ définie pour $0 \leq t \leq \infty$ a la forme (Lemay, 2001).

$$
Y(f)=\int_{0}^{\infty} \mathrm{y}(t) \exp (-j 2 \pi f t) d t
$$

Pour un signal continu défini par sa transformée de Fourier donnée par l'équation (1), en prenant une fréquence d'échantillonnage $f_{s}$ et un temps d'observation $\mathrm{T}$ on aura alors un échantillon $\mathrm{y}\left(t_{n}\right)$ de taille $N$. Cela revient à observer $\mathrm{y}(t)$ à des temps discrets espacés d'un pas $\Delta t=\frac{1}{f_{\mathrm{s}}} \quad$; on observe donc un signal aux instants $t_{n}=\mathrm{n} \times \Delta \mathrm{t}$ avec $0 \leq n \leq N-1$.
Ainsi, la transformée de Fourier discrète d'un signal donné s'exprime sous la forme suivante :

$$
\begin{gathered}
Y\left(f_{k}\right)=\Delta \mathrm{t} \sum_{\mathrm{n}=0}^{\mathrm{N}-1} \mathrm{y}_{\mathrm{n}} \exp \left(-\frac{j 2 \pi \mathrm{kn}}{\mathrm{N}}\right) \\
\text { avec } \mathrm{k}=0,1,2, \ldots, \frac{\mathrm{N}}{2}
\end{gathered}
$$

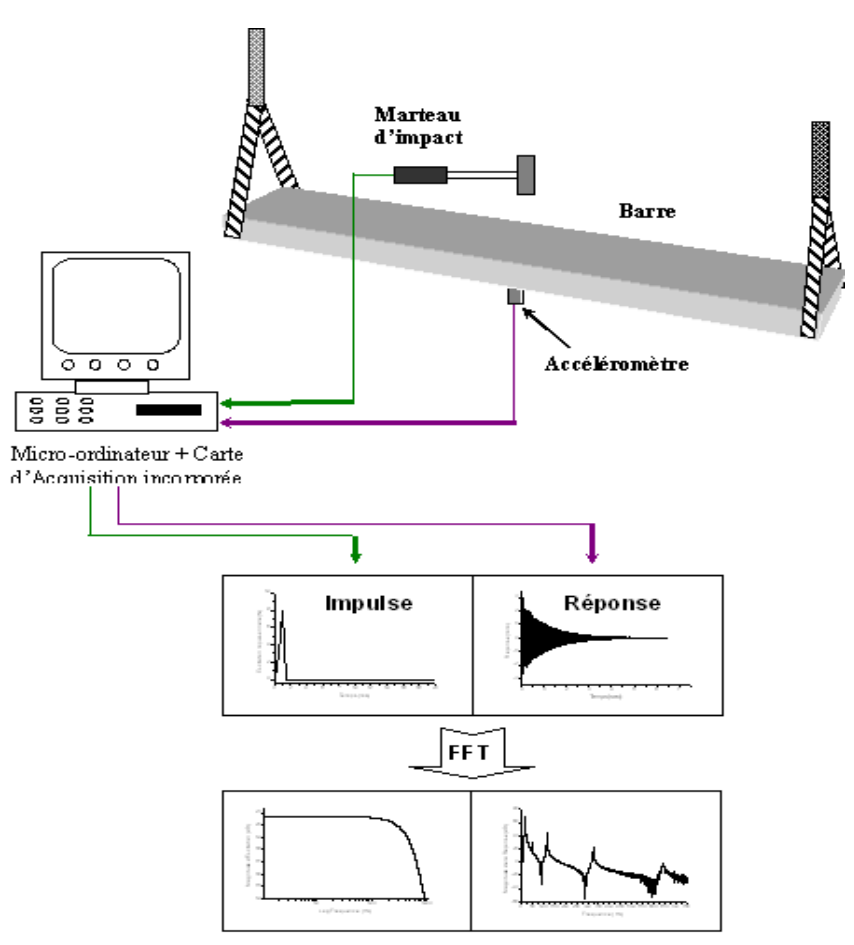

Fig.1 Procédure d'essai de vibration et acquisition de données pour le cas d'une barre libre aux extrémités

\subsection{Identification de module d'élasticité}

Dans la pratique, le calcul des fréquences de vibrations propres des systèmes ce fait sans tenir compte de la force d'amortissement, cela est dû au fait qui est généralement admis de négligé l'effet de l'amortissement sur les valeurs de la fréquence de vibration vue qu'il est faible par rapport à d'autres facteurs.

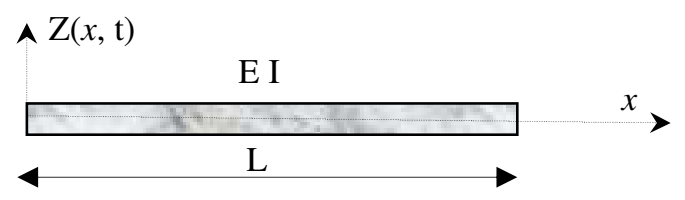

Pour une barre rectangulaire de section constante et de masse uniformément répartie et selon ses conditions géométriques aux extrémités, constitue un système vibrant que les équations du mouvement peuvent être résolues analytiquement par dérivation de l'équation de mouvement obtenue à partir d'une analyse de la 
seconde loi de newton pour les forces et les moments agissant sur la barre (Broch, 1984) :

$$
\rho \mathrm{S} \frac{\partial^{2} \mathrm{Z}(x, \mathrm{t})}{\partial \mathrm{t}^{2}}+\frac{\partial^{2}}{\partial x^{2}}\left(\mathrm{EI} \frac{\partial^{2} \mathrm{Z}(x, \mathrm{t})}{\partial x^{2}}\right)=0
$$

E : module de Young

I : moment d'inertie de la barre

$\rho$ : la masse volumique de la barre

$\mathrm{S}$ : la section de la barre

A partir de l'expression (3) on peut facilement aboutir a une forme d'écriture permettent de calculer le module de d'élasticité (module de Young) d'un matériau en fonction de la fréquence de résonance du système selon les conditions aux limites :

$$
\mathrm{E}=48 \pi^{2} \rho\left(\frac{\mathrm{L}^{2}}{\mathrm{~h}} \frac{f_{\mathrm{n}}}{\mathrm{K}_{\mathrm{n}}^{2}}\right)^{2} \quad \mathrm{~N} / \mathrm{m}^{2}
$$

$f_{\mathrm{n}}$ : fréquence de résonance de système.

$\mathrm{K}_{\mathrm{n}}$ : constante qui dépend des conditions aux limites de la barre.

\section{Procédure expérimentale}

Le micro-ordinateur gère, sous le contrôle d'une carte d'acquisition, la totalité du paramétrage du boîtier d'acquisition et du traitement de l'information recueillie à la suite de la percussion appliquée.

L'onde émise est transformée en signal électrique par un capteur de réponse (accéléromètre). Celui ci amplifie, filtre puis échantillonné par l'intermédiaire du boîtier d'acquisition. Puis, après conversion du signal analogique en information numérique, l'enregistrement est transféré directement via le port parallèle dans une mémoire utilisateur du PC. La composition spectrale de l'enregistrement est obtenue par (FFT) transformée de Fourier Rapide (fig.1).

Les matériaux qui ont été soumis à des tests expérimentaux sont des poutres en bois de Pin d'Alep et une poutre en acier.

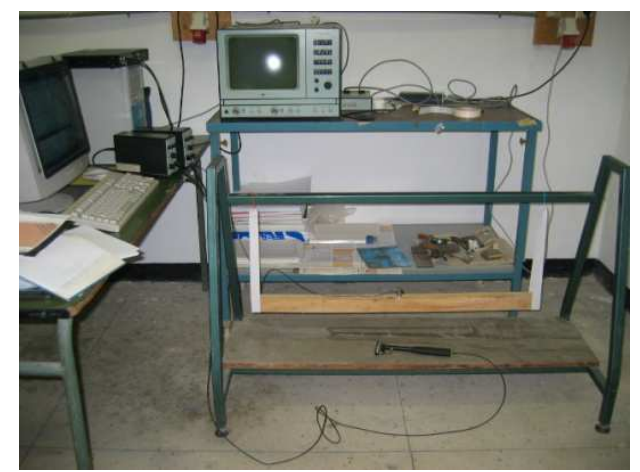

Fig.2. Installation d'équipements pour le cas d'une poutre en bois de Pin d'Alep libre à ses extrémités
Ces poutres en été placé de tel sort quelle constituent un système poutre libre sur ses deux extrémités (fig.1 \& 2).

\section{Présentation des résultats et discussion}

L'acquisition des données nous a permis l'identification des fréquences propre, ainsi que le module d'élasticité longitudinale de chaque mode de vibration des systèmes. En effet, nos systèmes ont été soumis à des excitations impulsionnelles de courte durée. Nous avons enregistré les réponses et les excitations.

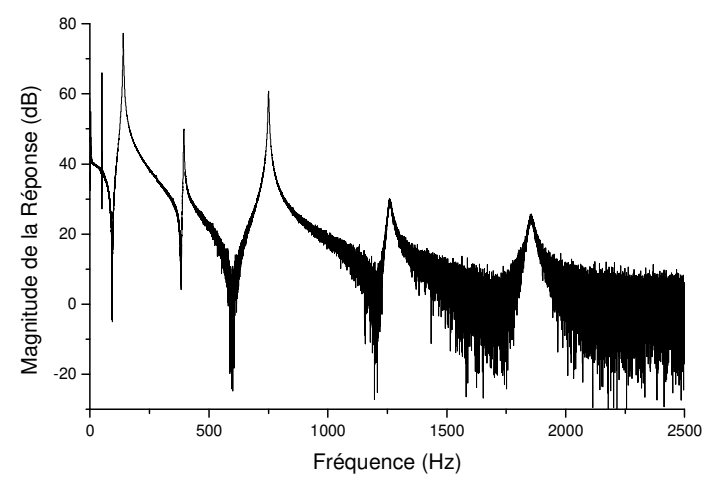

Fig.3. Spectre de réponse de la poutre sur ses deux extrémités

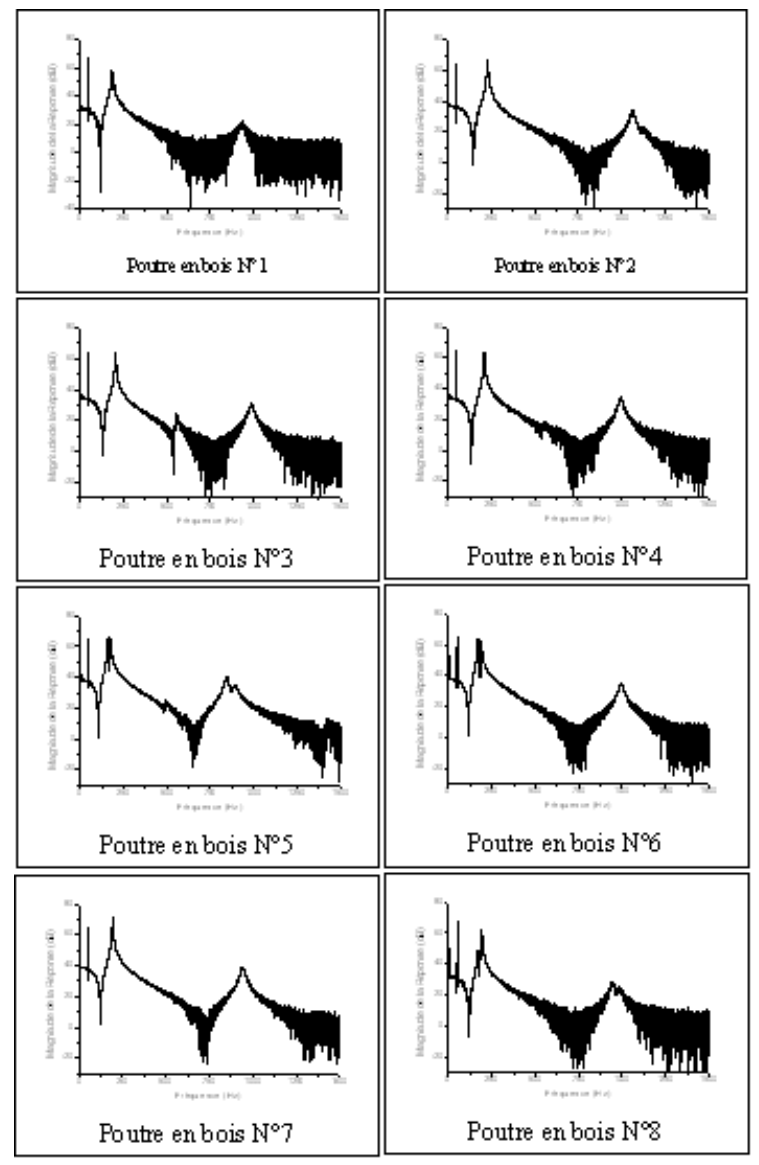

Fig.4. Spectre de réponse de la poutre en bois Pin d'Alep libre sur ses deux extrémités 


\begin{tabular}{|c|c|c|c|c|}
\hline Ptre & $\begin{array}{c}\text { Frq }(\mathrm{Hz}) \\
\mathrm{E}=10000 \\
\mathrm{MPa}\end{array}$ & $\begin{array}{c}\text { Frq }(\mathrm{Hz}) \\
\mathrm{E}=13000 \\
\mathrm{MPa}\end{array}$ & $\begin{array}{c}\text { Frq Exp } \\
(\mathrm{Hz})\end{array}$ & $\begin{array}{c}\text { E ident. } \\
(\mathrm{MPa})\end{array}$ \\
\hline 1 & 633.28 & 631.38 & 753.37 & 9400 \\
\hline 2 & 616.67 & 615.47 & 607.00 & 13600 \\
\hline 3 & 609.16 & 609.16 & 677.59 & 10700 \\
\hline 4 & 635.97 & 635.78 & 790.91 & 12300 \\
\hline 5 & 631.95 & 631.67 & 788.65 & 8600 \\
\hline 6 & 627.32 & 625.89 & 730.26 & 9800 \\
\hline 7 & 607.00 & 607.00 & 705.99 & 9800 \\
\hline 8 & 620.18 & 619.93 & 718.86 & 9900 \\
\hline
\end{tabular}

résonance obtenues en admettant une gamme de modules d'élasticité allant de 10000 à 13000 Mpa.

Tableau 1. Valeurs des fréquences de résonance et de module d'élasticité de flexion de premier mode de vibration pour le cas des poutres en bois de Pin d'Alep.

L'exploitation des valeurs de fréquences de résonance expérimentale a permit le calcul du module d'élasticité pour les poutres de Pin d'Alep. Les valeurs obtenues à l'issue de ce calcul convergent avec celles théoriques (Fig.5).

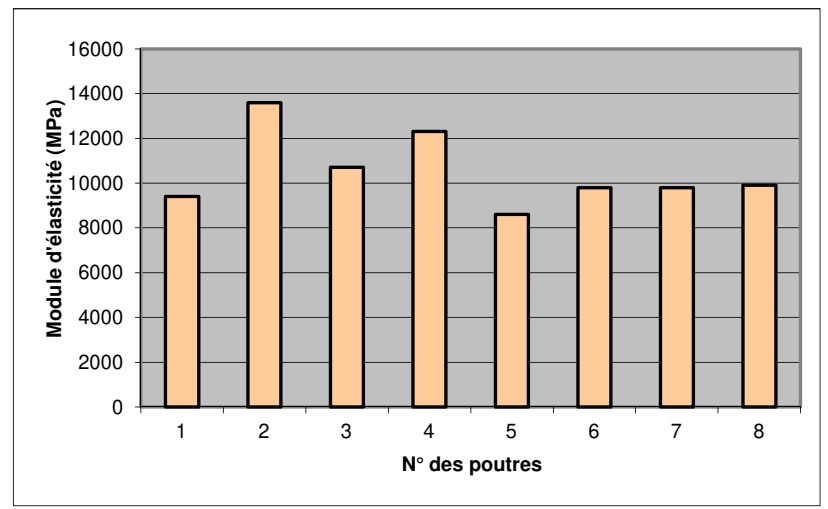

Figure 5 Distribution des modules d'élasticité des poutres en bois de Pin d'Alep

Les graphes obtenus par acquisition, correspondant aux spectres de réponses pour chaque système sont représentés par les figures $3 \& 4$. Ils correspondent aux transformées de Fourier dans le domaine de fréquence des réponses temporelles. Les fréquences de résonance des modes de vibration sont clairement identifiées et correspondent aux pics présents sur ces spectres. Aussi on peut remarquer que le bruit est prédominant, ce qui empêche l'apparition d'autres modes de vibration.

En effet, pour le cas d'un marteau d'impact, la gamme de fréquence de l'excitation fournie par ce dispositif est déterminée par la rigidité des surfaces de contact entre marteau-poutre et la masse de la tête de marteau.

Après cette gamme, les autres modes de vibration de la structure ne seront pas excités (il n y a pas assez d'énergie pour l'excitation. Ce qui explique la raison pour laquelle nous avons obtenu plus de pics de résonance qui ont été clairement identifiés.

Dans le tableau-1, nous avons présenté les valeurs de fréquence de résonance obtenues à partir des spectres de réponse pour chaque système. Nous avons présenté les valeurs des 4 premiers modes de vibration. L'analyse de ces valeurs, nous a permis d'identifier le module d'élasticité longitudinal de nos éprouvettes.

Par ailleurs, pour estimer la plage des fréquences de résonance du bois de pin d'Alep, nous avons injecté la valeur statique du module d'élasticité de notre matériau. Nous pouvons noter que les valeurs théoriques et celles obtenues expérimentalement vont dans le même ordre.

Pour le cas des poutres en bois de Pin d'Alep, nous présentons (Tab.1) l'ensemble des valeurs expérimentales des fréquences de résonance du premier mode de vibration pour chaque poutre, ces valeurs ont été comparées avec les valeurs théoriques de fréquence de

Dans les bois, la répartition de la masse volumique est généralement variable dans les trois sens (radial, tangentiel et longitudinal), et c'est le cas de nos 08 poutres (effet d'hétérogénéité). Cette remarque a été confirmée par la différence des valeurs de module d'élasticité entre ces poutres. Ces différentes valeurs se situent quand même autour de la gamme que peut avoir le bois et notamment le Pin d'Alep.

\section{Conclusion}

Le but de ce présent travail était de proposer une démarche expérimentale permettant l'identification du module d'élasticité existant dans le matériau. Les résultats expérimentaux obtenus ont montré la concordance des données qui restent en général proches de celles données par les normes aussi bien pour le cas de l'acier que du bois. Au terme de cette intéressante investigation, il y a lieu de noter qu'a travers cette méthode, nous avons pu aboutir a démontrer le caractère variable de module d'élasticité (phénomène reconnu pour le cas des bois). Nous avons utilisé un même type de bois pour remarquer qu'il y avait une différence significative autour de la valeur de module d'élasticité entre ces bois, mais qui reste cependant corrélée avec la gamme que peut avoir ce type de bois.

Par ailleurs, nous avons observé la variabilité du module d'élasticité en fonction du mode de vibration pour le cas de la poutre en acier. Cependant, on n'a pas pu identifier d'autres modes de vibration pour calculer le module d'élasticité pour le cas des poutres en bois. Ceci est du probablement à la nature de la surface de contact entre le marteau d'impact et le bois. Nous pensons que la surface 
n'est pas aussi rigide pour permettre l'apparition d'autres modes de vibration.

\section{References}

[1]-Broch J.T. (1984). Mechanical vibration and shock measurements. Brüel\&Kjaer. $2^{\text {nd }}$ Edition.

[2]-Dumas J, Bennevault B. (2001). Debuter la mesure vibratoire. MVI technologies group. Version Février

[3]- DØssing O. (1987a). Structural Testing Part 1: Mechanical Mobility Measurements. Brüel \& Kjaer, April.

[4]-D Øssing O. (1988b). Structural Testing Part 2 : Modal Analysis and Simulation. . Brüel \& Kjaer, March.

[5]-Ewins D.J. (1984). Modal Testing: Theory and Practice. Research Studies Press, Ltd, England.

[6]-Haines D., Leban J.M. (1997). Evaluation of the MOE of Norway spruce by the resonance flexure method. Forest Products Journal, Vol.47 n ${ }^{\circ} 10$, p 9094.

[7]-Lemay J. (2001). Acquisition, traitement de données, cours GMC-64506. Université LAVAL. Septembre

[8]-Zaveri K, Phil M. (1984). Modal Analysis of Large Structures-Multiple Exciter Systèms. Brüel \&Kjaer, $1^{\text {st }}$ edition, November 